\title{
Design of 500MHz Wideband RF Front-end
}

\author{
Zhengqing Liu, Zhiqun $\mathrm{Li}^{+}$ \\ Institute of RF- \& OE-ICs, Southeast University, Nanjing, 210096; \\ School of Integrated Circuits, Southeast University, Nanjing, 210096; \\ ${ }^{+}$Corresponding author: zhiqunli@seu.edu.cn
}

\begin{abstract}
This paper presents a design of $500 \mathrm{MHz}$ wideband RF front-end for P-band radar based on $0.18 \mu \mathrm{m} \mathrm{RF}$ process. The front-end works at the center frequency of $500 \mathrm{MHz}$ with $200 \mathrm{MHz}$ bandwidth, which transforms single-end signal to differential signal, amplifies it and mixes it with local oscillator signal, outputting an IF signal of $10 \pm 5 \mathrm{MHz}$. It adopts noise-canceling to lower the noise figure. It adopts a common-gate input mode to achieve good input match. The post-simulation results indicate that under a $3.3 \mathrm{~V}$ power supply, the $\mathrm{S} 11$ is less than $-20 \mathrm{~dB}$ in the work frequency band, the conversion voltage gain is $13.5 \mathrm{~dB}$. A $\mathrm{NF}$ of $8.6 \mathrm{~dB}$ and input $1 \mathrm{~dB}$ compression point of $-8.5 \mathrm{dBm}$ are achieved with a total current consumption of $11 \mathrm{~mA}$.
\end{abstract}

Key words: RF front-end; noise canceling; high linearity

\section{Introduction}

Wideband radio receivers have recently drawn significant research interests. Co-operability with other communication devices (e.g., cellular, WLAN) operating in the same spectrum is mandatory, setting especially stringent demands on the wideband linearity of such a receiver. A single-ended RF input avoids the use of an external broadband balun and its accompanying losses. RF front-end module is shown in Fig. 1. The RF signal is mixed at the mixer with the local oscillation signal to obtain the desired intermediate frequency signal $(10 \pm 5 \mathrm{MHz})$.

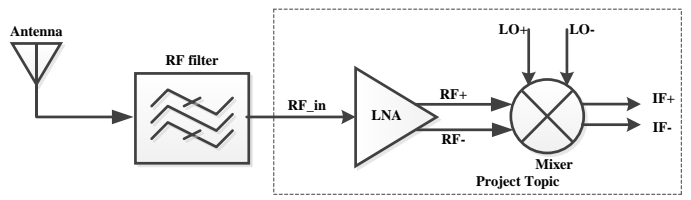

Fig.1 Block diagram of RF front end 
Placed at the front end of the entire receiver, the noise of front end has huge impacts on the receiving circuit. Thus it must have certain gain while not introduce too many noises. We should also consider the linearity requirements as the signal is amplified (usually less than $30 \mathrm{~dB}$ ). We may see significant compression at the output of the mixer. The front end should achieve low noise factor, high linearity, low power consumption and adequate voltage gain.

\section{Circuits Design}

\subsection{Proposed balun LNA topology}

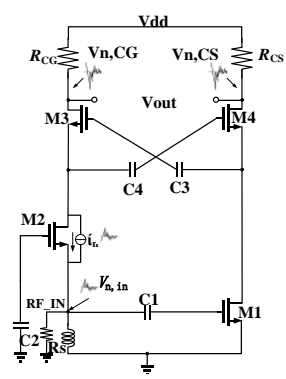

Fig.2 Topology of the proposed LNA

Fig. 2 shows a balun-LNA consisting of a parallel operating common gate (CG) and common source (CS) stage. The CG stage realizes wideband input matching and in-phase gain, while the CS stage realizes an anti-phase output signal. In a CG stage only, the noise of the CG transistor would be dominant when the input impedance is matched to $R_{S}\left(R_{S}=1 / g_{m, C G}\right)$. However, using a properly designed CS stage this noise ( $i_{n}$ in Fig. 2 ) can be canceled. The noise current $\left(i_{n}\right)$ generates a noise voltage $\left(v_{n, i n}\right)$ on the source resistor and a larger voltage in anti-phase across $\mathrm{R}_{\mathrm{CG}}\left(v_{n, C G}\right)$. The input noise voltage is amplified by the CS stage to $v_{n, C S}$, which is in-phase and fully correlated with $v_{n, C G}$. For equal CG and CS stage gain, the noise due to the CG transistor is fully canceled at the differential output, while the signal contributions to the output signal add up to create a balanced output[1]. The parallel CS-CG topology (or balun-LNA) cancels the noise of the CG transistor in order to obtain a noise figure (NF) close to or lower than $3 \mathrm{~dB}$.

The CG stage is biased using an external inductor to obtain low-noise operation and save voltage headroom. The inductor can cancel the capacitive part of input 
impedance, making the input impedance purely resistive at the frequency of internet. By using the inductor, the input impedance can be perfectly matched to $\mathrm{R}_{\mathrm{S}}(50 \Omega)$ at the frequency of $500 \mathrm{MHz}$ and experiences little fluctuation within the whole band, achieving a good input matching.

The cross-coupled capacitor network can be used for compensating the gain and phase mismatch. This network also can lower the noise contribution of the cascode transistors M3 and M4 by the $g_{\mathrm{m}}$-boosting effect. In simulation, the cross-coupled capacitors can lower the noise figure by $0.5-0.9 \mathrm{~dB}$ [2].

To totally cancel the noise generated by M2, the transistors and resistors should satisfy the following formula [3]:

$$
g_{m, M 2} \times R_{C G}^{2}=g_{m, M 2} \times R_{s}^{2} \times g_{m, M 1}^{2} \times R_{C S}^{2} .
$$

To achieve input matching and balanced output at the drain of the cascode transistors, the devices in the topology should also meets this formula:

$$
g_{m, C G} \times R_{C G}=g_{m, C S} \times R_{C S}=\frac{A_{V}}{2} \quad R_{S}=\frac{1}{g_{m, C G}} .
$$

$R_{S}$ is usually equal to $50 \Omega$, making the conductance of M2 20ms. Choose the sizes and bias voltage according to the formula above, the noise of CG transistor can be totally cancelled, making no contribution to the total NF. Since the CG noise is cancelled, the CS noise dominates in the LNA. The NF of CS stage can be expressed as

$$
\mathrm{F}=1+\frac{\gamma}{\alpha} \cdot \frac{1}{g_{m} \cdot R_{S}}
$$

To reduce the noise generated by M1 of the CS stage, we choose $\mathrm{g}_{\mathrm{m}, \mathrm{CS}}$ of M1to be 4 times larger than $\mathrm{g}_{\mathrm{m}, \mathrm{CG}}$, then $R_{C G}=4 R_{C S}$ [4]. Thus the equilibrium noise voltage at the gate of it can be greatly reduced, and so is the total NF of the topology. By configuring the sizes of the devices of the topology like this, we simultaneous achieve output balancing and noise-canceling at the drains of transistors M3, M4. In simulation, a NF of 2.7-2.8 dB is achieved within the band, proving the excellent performance of the proposed balun LNA.

As derived above, not only the noise of the impedance matching device is canceled, but also its nonlinearity. Assume the nonlinear behavior of M1 can be modeled be the drain-source current which depends nonlinearly on both voltage variations $v_{g s}$ and $v_{d s}$ around their DC bias points. The source signal $v_{s}$ causes a nonlinear drain-source current $i_{d s}$ which is converted into a nonlinear voltage $v_{i n}$ at the input via the (linear) source resistor. The nonlinear input voltage $v_{\text {in }}$ can be 
written as

$$
v_{i n}=a v_{S}+v_{N L},
$$

where the $\alpha$ represents Taylor coefficient and $v_{N L}$ contains all unwanted nonlinear terms. The output voltage of the CG-stage and CS-stage can be written as

$$
v_{\text {out }, C G}=\left((1-\alpha) \cdot v_{S}-v_{N L}\right) \cdot \frac{A_{V}}{2}, \quad v_{\text {out }, C S}=-\left(\alpha \cdot v_{S}+v_{N L}\right) \cdot \frac{A_{V}}{2} \text {. }
$$

After subtraction only the linear signal remains

$$
v_{\text {out }, \text { diff }}=v_{\text {out }, C G}-v_{\text {out }, C S}=v_{S} \cdot A_{V} \text {. }
$$

In conclusion, all noise and distortion currents generated by the CG-transistor can be canceled, irrespective of whether produced due to nonlinearity of the transconductance or nonlinearity of the output conductance.

\subsection{Design of RF Front-end}

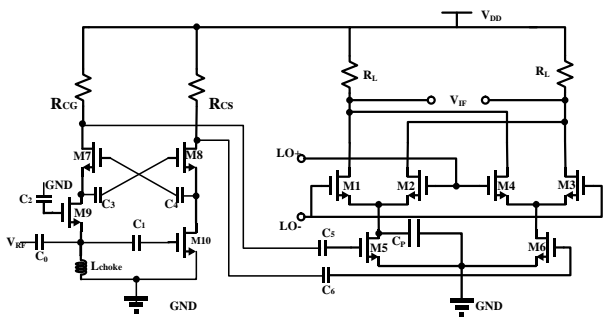

Fig.3 Schematic of the front end

In Fig. 3, the principle of RF front-end topology is shown. The output of balun LNA is connected to the Gilbert double balanced mixer by direct coupling. The differential signal is interposed on the gate of transforming transistor of the mixer, mixed with the local oscillator and converted to differential IF signal at the drains of switching transistors M1-M4.

\subsubsection{Bandwidth Analysis}

There are seven RF signal nodes in the circuit shown In Fig. 3, which are the signal inputting node, the drains of CG and CS transistors, output nodes of balun LNA, the drains of transconductance transistors of the mixer. The real parts of impedances of cascode transistors of balun LNA and the switching transistors of the mixer are very small seen from the source. These nodes don't limit the overall bandwidth. Under the condition of input matching, though accompanied by large amounts of parasitic capacitances, the real part of input impedance is only equal 
to $50 \Omega$. The impedances of output nodes of the balun LNA are relatively high than other nodes ( $\mathrm{R}_{\mathrm{CS}} 100 \Omega$ and $\mathrm{R}_{\mathrm{CG}} 400 \Omega$ respectively), making the pole at these two nodes dominant. The choice of the transconductance transistors of the mixer should be careful to avoid introduction of large parasitic capacitances, thus not decreasing the bandwidth significantly. The simulation indicates that, in our design of $500 \mathrm{MHz} R F$ front end, the input matching coefficient S11 is less than $-20 \mathrm{~dB}$ from $400 \mathrm{MHz}$ to $600 \mathrm{MHz}$ and is less than $-10 \mathrm{~dB}$ within a bandwidth of $400 \mathrm{MHz}$ around the center frequency; the conversion gain changes only a little within the band and will decrease fiercely only when input signal frequency is down below $300 \mathrm{MHz}$, which proves the good performance of the topology.

\subsubsection{Noise Analysis}

To simplify the calculation, transistors are assumed to have infinite output impedance and the bias current source of the CG-transistor is assumed to be ideal. Furthermore only the thermal noise of the resistors and of the transistors is taken into account. The noise factor of balun LNA can be expressed as [4]

$$
\mathrm{F}=1+\frac{\gamma \cdot g_{m, C G} \cdot\left(R_{C G}-R_{C S} \cdot g_{m, C S} \cdot R_{S}\right)^{2}}{R_{s} \cdot A_{V}{ }^{2}}+\frac{\gamma \cdot g_{m, C S} \cdot R_{C S}^{2}\left(1+g_{m, C G} \cdot R_{S}\right)^{2}}{R_{S} \cdot A_{V}{ }^{2}}+\frac{\left(R_{C G}+R_{C S}\right) \cdot\left(1+g_{m, C G} \cdot R_{S}\right)^{2}}{R_{S} \cdot A_{V}{ }^{2}},
$$

where the second part is the contribution of the CG transistor, which can be totally cancelled with adequately designed dimensions of the devices of balun LNA, making the third part dominant in the total noise factor. $A_{V}$ denotes the total voltage gain of the balun LNA, equaling

$$
A_{V}=g_{m, C G} \cdot R_{C G}+g_{m, C S} \cdot R_{C S} .
$$

The third part can be expressed as

$$
F_{C S}=\frac{4 \cdot \gamma \cdot g_{m, C S} \cdot R_{C S}^{2}}{R_{S} \cdot g_{m, C S} \cdot R_{C S}^{2}}=\frac{4 \cdot \gamma}{R_{S} \cdot g_{m, C S}} .
$$

We can see, by improving the transconductance of CS transistor, the noise factor of the topology can be further optimized.

The noise of the mixer stage is complicated. Every device in the circuit contributes some to the total output noise. Of all the noise sources, the thermal noise and flick noise are the most important [5]. But the flick noise is only prominent at the frequency down below corner frequency $f_{\mathrm{C}}$, equaling

$$
f_{C}=\frac{\mathrm{K}}{4 \kappa T} \omega_{T},
$$


which is approximately from $500 \mathrm{kHz}$ to $5 \mathrm{MHz}$, where $\mathrm{K}$ is a process-related coefficient. So the flick noise is not important in this design (with an IF at 10 $\mathrm{MHz}$ ). In our analysis of the noise factor of the mixer, this part is ignored, assuming abrupt LO transition with a 50\% duty cycle at the same time. The equivalent noise at the input of the mixer can be expressed as [6]

$$
\overline{V_{n, i n}^{2}}=2 \pi^{2}\left(\frac{c_{P}^{2} \cdot \omega^{2}}{g_{m, S}^{2}}+1\right) \cdot k T \cdot\left(\frac{\gamma}{g_{m, C}}+\frac{\gamma \cdot C_{P}^{2} \cdot \omega^{2}}{g_{m, S} \cdot g_{m, C}^{2}}+\frac{2}{g_{m, C}^{2}}\right),
$$

where the $\mathrm{g}_{\mathrm{m}, \mathrm{S}}$ and $\mathrm{g}_{\mathrm{m}, \mathrm{c}}$ denote the transconductance of switching transistors and transforming transistors of the mixer respectively, $C_{P}$ is the parasitic capacitance at node P, as shown in Fig. 3. We can judge from the formula that the existence of $\mathrm{C}_{\mathrm{P}}$ is the reason why the noise of switching transistors would contribute to the output noise. According to the equation (11), we should configure the circuit to minimize the $\mathrm{C}_{\mathrm{P}}$ and maximize $\mathrm{g}_{\mathrm{m}, \mathrm{C}}$, thus optimizing the noise characteristic. However, the $\mathrm{g}_{\mathrm{m}, \mathrm{C}}$ experiences a tradeoff with linearity requirements and is limited by the power consumption. As we know

$$
I P_{3} \propto V_{G S}-V_{T H}, \overline{v_{n, i n}^{2}} \propto \frac{4 k T_{Y}}{g_{m}}=\frac{4 k T \gamma}{2 I_{D}}\left(V_{G S}-V_{T H}\right) .
$$

The dimensions of the transistors and the bias voltage should balance the power consumption, noise performance and linearity. The simulation shows, the noise figure of the mixer is $12-13 \mathrm{~dB}$, and the $1-\mathrm{dB}$ compression point is around $-3 \mathrm{dBm}$ with respect to a $\mathrm{R}_{\mathrm{S}}$ of $300 \Omega$, which would not deteriorate the linearity of the front end.

\section{The post-simulation results}

This paper presents a wideband RF front end for 400 600MHz P-band radar applications in $0.18 \mu \mathrm{m}$ RF CMOS process. It consumes about $11 \mathrm{~mA}$ current from $3.3 \mathrm{~V}$ power supply. The layout of the LNA is shown in Fig. 4. The post-simulation result of NF is shown in Fig.5. The NF equals 8.6 8.8dB. The post-simulation of S11 is shown in Fig.6. During 400 600 MHz, S11 is -23 -20 $\mathrm{dB}$; the post-simulation of conversion gain is shown in Fig.7. The conversion gain is about $12.7 \mathrm{~dB}$ with $0.2 \mathrm{~dB}$ fluctuation when the IF frequency changes between $5 \mathrm{MHz}$ and $80 \mathrm{MHz}$; the post-simulation of $\mathrm{IP}_{\mathrm{ldB}}$ is shown in Fig.7. $\mathrm{IIP}_{\mathrm{IdB}}$ is -8.5 $\mathrm{dBm}$. The $\mathrm{IP}_{\mathrm{ldB}}$ is achieved by date fitting on Origin using the date obtained in post-simulation. 


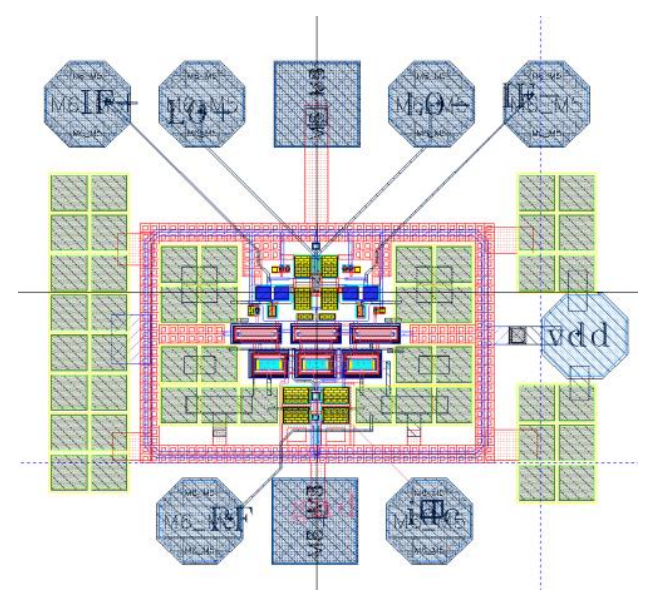

Fig. 4 Layout of the LNA

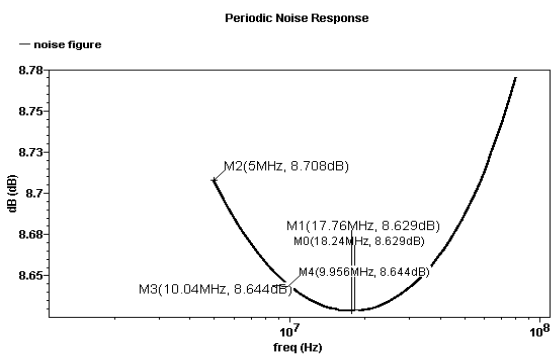

Fig.5 Post-simulation result of NF

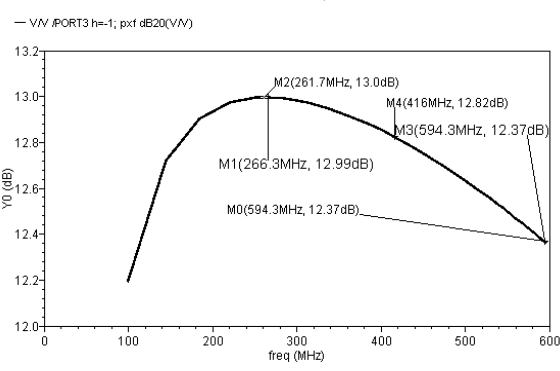

Fig.7 Post-simulation of conversion gain

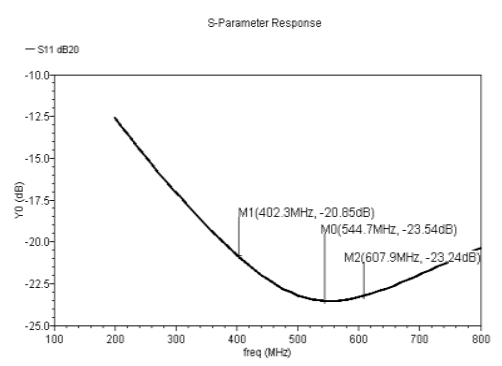

Fig.6 Post-simulation result of S11

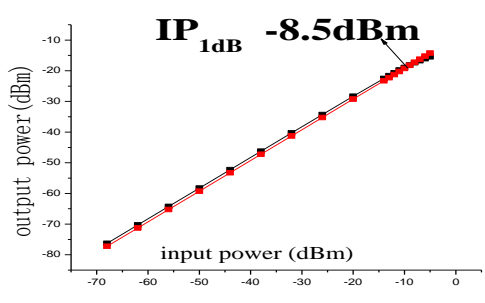

Fig.8 Post-simulation of $\mathrm{IP}_{1 \mathrm{~dB}}$ 
The overall post-simulation result is shown below:

\begin{tabular}{ll}
\hline Parameter & value \\
\hline Supply voltage $(\mathrm{V})$ & 3.3 \\
Power consumption $(\mathrm{mW})$ & 36.1 \\
$\mathrm{~S} 11(\mathrm{~dB})$ & $-25 \sim-20$ \\
Conversion gain $(\mathrm{dB})$ & $12.8 \sim 13.2$ \\
$\mathrm{NF}(\mathrm{dB})$ & $8.6 \sim 8.7$ \\
$\mathrm{IP}_{1 \mathrm{~dB}}(\mathrm{dBm})$ & -8.5 \\
\hline
\end{tabular}

\section{Summary}

This paper presents a wideband RF front end for $400 \sim 600 \mathrm{MHz}$ P-band radar applications in $0.18 \mu \mathrm{m}$ RF CMOS process. The front end adopts linearity enhancement, noise cancellation to decrease the NF and improve the linearity. Post-simulation results show that LNA can be fully adapted to P-band radar system applications.

\section{References}

[1] Blaakmeer, S. C.; Klumperink, E. A. M.; Leenaerts, D. M. W.; Nauta, B., " The Blixer, a Wideband Balun-LNA-I/Q-Mixer Topology," Solid-State Circuits, IEEE Journal of , vol.43, no.12, pp.2706,2715, Dec. 2008

[2] Sherif A. Saleh, Maurits Ortmanns, and Yiannos Manoli. A Low-Power Differential Common-Gate LNA. IEEE Conferences.10.1109/MWSCAS.2008.4616755,2008.

[3] Donggu Im; Ilku Nam; Seong-Sik Song; Hong-Teuk Kim; Kwyro Lee, "A CMOS resistive feedback single to differential low noise amplifier with multiple-tuner-outputs for a digital TV tuner," Radio Frequency Integrated Circuits Symposium, 2009. RFIC 2009. IEEE, vol., no., pp.555, 558, 7-9 June 2009 doi: 10.1109/RFIC.2009.5135602.

[4] Blaakmeer, S. C.; Klumperink, E. A. M.; Leenaerts, D. M. W.; Nauta, B., "Wideband Balun-LNA With Simultaneous Output Balancing, Noise-Canceling and Distortion-Canceling," Solid-State Circuits, IEEE Journal of , vol.43, no.6, pp.1341,1350, June 2008

[5] Darabi, H.; Abidi, A. A., "Noise in RF-CMOS mixers: a simple physical model," Solid-State Circuits, IEEE Journal of , vol.35, no.1, pp.15,25, Jan. 2000doi: 10.1109/4.818916

[6] Behzad Razavi," RF Microelectronics, Second Edition," Publishing House of E- lectronics Industry, pp. 381-383, August 2012 